\title{
Sérologie du SRAS-CoV-2 (COVID-19) : Répercussions sur la pratique clinique, la médecine de laboratoire et la santé publique
}

\author{
Paul Van Caeseele MD, pour le Réseau des laboratoires de santé publique du Canada; Dana Bailey MSc PhD, \\ pour la Société canadienne des clinico-chimistes; Sarah E. Forgie MD MÉd, pour l'Association pour la microbiologie \\ médicale et l'infectiologie Canada; Tanis C. Dingle PhD, pour l'Association canadienne de microbiologie clinique et \\ des maladies infectieuses; Mel Krajden MD, pour le Groupe de travail sur l'immunité face à la COVID-19
}

\begin{abstract}
— Citation : CMAJ 2020 August 24;192:E973-9. doi : 10.1503/cmaj.201588-f; publication hâtive le 4 août 2020
Voir la version anglaise de l'article ici : www.cmaj.ca/lookup/doi/10.1503/cmaj.201588

Une liste complète des membres des 5 groupes d'auteurs est présentée à la fin de l'article.
\end{abstract}

C hez les personnes ayant contracté le coronavirus du syndrome respiratoire aigu sévère 2 (SRAS-CoV-2), le tableau clinique varie de l'absence de symptômes à la détresse respiratoire gravissime qui accompagne la maladie à coronavirus 2019 (COVID-19)1. À l'heure actuelle, le diagnostic des infections aiguës ou nouvelles au SRAS-CoV-2 repose en général, sur le dépistage moléculaire de l'ARN viral dans des spécimens provenant des voies respiratoires supérieures et inférieures recueillis dans les 2 à 7 jours suivant une exposition $^{2,3}$. Durant cette période, l'excrétion virale est active et les personnes infectées peuvent transmettre le virus. Même si l'ARN viral est encore détecté dans les spécimens de sécrétions respiratoires et de selles chez certaines personnes pendant plusieurs semaines après leur rétablissement, cela ne semble pas poser un risque de transmission ${ }^{4,5}$. Les tests sérologiques reposent sur le dépistage des anticorps dirigés contre le SRAS-CoV-2 dans le sang, le sérum ou le plasma. Le rôle de la sérologie dans le diagnostic de la COVID-19 aiguë est limité parce qu'il faut habituellement attendre au moins 7 à 14 jours, voire plus, après le déclenchement des symptômes pour obtenir un titre d'anticorps anti-SRAS-CoV-2 mesurable et fiable ${ }^{6,7}$. Mais on s'est intéressé à l'application potentielle des tests sérologiques à des fins aussi diverses que l'autorisation des voyages internationaux, la stratification du risque de réinfection en milieu de travail et la réduction de l'anxiété du public dans le but de faciliter la reprise de l'activité économique ${ }^{8,9}$. Nous passons en revue ce que l'on sait actuellement sur les tests sérologiques pour le SRAS-CoV-2, soit un corpus de données scientifiques et cliniques en constante évolution (encadré no 1); nous en mesurons l'impact sur les soins cliniques, le développement des services requis et l'interprétation des tests; et nous formulons un avis sur leur utilisation appropriée à des fins cliniques et de santé publique.

\section{POINTS CLÉS}

- Plusieurs tests sérologiques commerciaux servant au dépistage des anticorps anticoronavirus du syndrome respiratoire aigu sévère 2 (SRAS-CoV-2) ont été approuvés par Santé Canada, et certains fabricants les disent dotés d'une sensibilité de $95 \%$ et d'une spécificité d'environ 99,5\%.

- Il n'a pas encore été démontré que la présence d'anticorps antiSRAS-CoV-2 détectables confère une immunité significative ou durable contre la réinfection. C'est pourquoi les tests sérologiques ne devraient pas guider les décisions individuelles relatives à l'exposition personnelle ou professionnelle, à l'utilisation de l'équipement de protection personnelle et à la distanciation physique.

- À l'heure actuelle, les indications cliniques des tests sérologiques dans les établissements de santé sont limitées et le dépistage sérologique du SRAS-CoV-2 n'a aucun impact sur la prestation des soins cliniques de routine.

- Pour l'instant, les tests sérologiques devraient servir principalement à la recherche concernant l'immunité contre le SRAS-CoV-2 et à des études de population pour éclairer les réponses de la santé publique face à l'épidémie de maladie à coronavirus 2019 (COVID-19) au Canada.

\section{Quelles sont les réponses immunitaires au SRAS-CoV-2?}

Le génome du SRAS-CoV-2 encode 4 protéines structurelles principales: la glycoprotéine de surface ou de spicule (S), l'enveloppe, la membrane et la nucléocapside $(\mathrm{N})^{10}$. Les tests sérologiques actuels détectent les anticorps dirigés contre les épitopes des protéines structurelles $\mathrm{S}$ ou $\mathrm{N}$.

Les spicules de surface forment la « couronne » observée à la micrographie électronique des coronavirus; elles jouent un rôle 


\section{Encadré no 1 : Données utilisées pour la présente revue}

Nous avons interrogé PubMed pour recenser les articles publiés en langue anglaise entre le $1^{\text {er }}$ janvier et le 30 juin 2020 sur le coronavirus du syndrome respiratoire aigu sévère 2 (SRAS-CoV-2), la réponse immunitaire, le diagnostic et la sérologie à partir des termes médicaux suivants en langue anglaise : coronavirus disease 2019 (COVID-19), antibodies, serology, serologic test, diagnosis, point-of-care testing, immunoglobulin, immunoassay, enzyme-linked immunosorbent assay, immunity, humoral, crossreaction, neutralization assay et multisystem inflammatory syndrome in children.

Nous avons recensé d'autres articles en procédant à des interrogations hebdomadaires de la base de données LitCOVID sous les rubriques mécanisme et diagnostic entre le 15 mars et 30 juin 2020.

Nous avons en outre identifié des études pertinentes en consultant des experts canadiens et en parcourant les prépublications sur la COVID-19 et le SRAS-CoV-2 de medRxiv et bioRxiv. De plus, nous avons régulièrement consulté les lignes directrices et ressources connexes émanant d'organisations internationales, telles que l'Organisation mondiale de la santé, Santé Canada, l'Agence de la santé publique du Canada, la US Food and Drug Administration, les US Centers for Disease Control and Prevention et l'Infectious Diseases Society of America, sur les conseils des organismes canadiens participants.

crucial dans la pathogenèse virale, c'est pourquoi les études se sont concentrées sur les anticorps dirigés contre des parties spécifiques de la glycoprotéine $\mathrm{S}$. Chaque protéine de spicule se compose de 2 sous-unités : la sous-unité $\mathrm{S} 1$ se lie aux récepteurs de l'enzyme de conversion de l'angiotensine 2 sur les cellules de plusieurs organes par l'entremise de son domaine de liaison au récepteur; et la sous-unité S2 médie la fusion entre le virus et la membrane cellulaire de l'hôte. Le domaine de liaison au récepteur de la protéine $S$ est une importante cible vaccinale et thérapeutique parce qu'une catégorie d'anticorps ciblant le domaine de liaison au récepteur semble bloquer la liaison virale et neutraliser l'infectivité virale in vitro ${ }^{11,12}$.

La figure 1 illustre le mode de réponse immunitaire au SRASCoV-2. Même si on considère généralement que les anticorps IgM (immunoglobuline M) et IgA (immunoglobuline A) apparaissent tôt dans la plupart des infections virales aiguës, on ignore si c'est le cas avec le SRAS-CoV- ${ }^{13}$. Dans la COVID-19, comme dans le SRAS et le syndrome respiratoire du Moyen-Orient (SRMO), les taux d'anticorps IgM et IgG semblent tous deux détectables environ 2 à 3 semaines après l'apparition des symptômes ou l'exposition $n^{6,714,15}$. Toutefois, dans certains cas légers et asymptomatiques, les anticorps peuvent être indétectables, du moins à l'intérieur de la période à laquelle certaines études récentes font référence $(<46 \mathrm{j})^{15-17}$.

Les tests commerciaux disponibles ciblent 1 ou plus des 3 isotypes d'anticorps (c.-à-d., IgA, IgM ou IgG), ou toutes les immunoglobulines. Les 2 principaux types de tests commerciaux sont décrits dans l'encadré no 2 . Une liste à jour des tests diagnostiques cliniques approuvés pour les anticorps antiSRAS-CoV-2 est disponible sur le site Web de Santé Canada20. Même si plusieurs immunodosages en laboratoire ont été approuvés, les données probantes sont insuffisantes pour

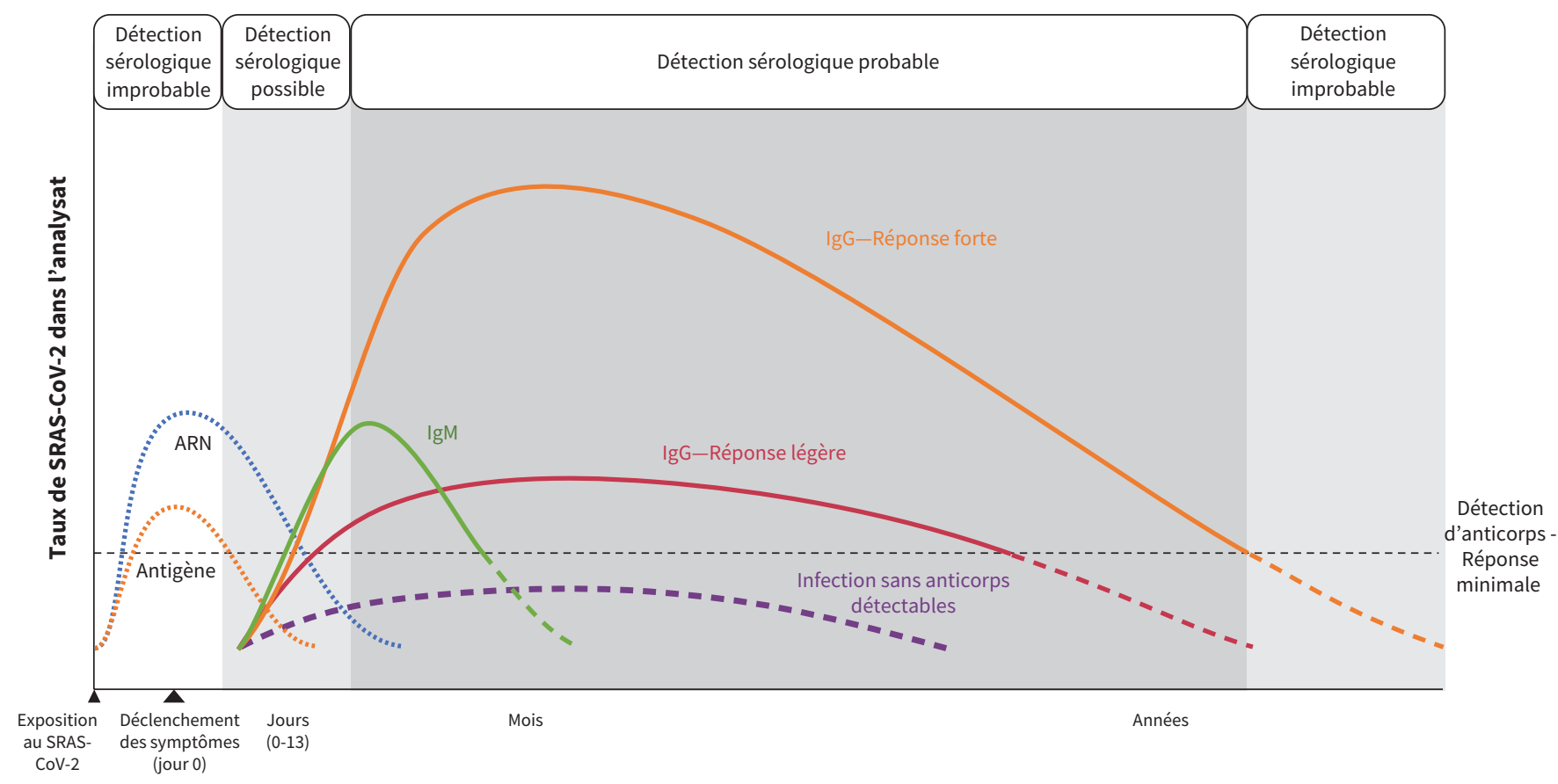

Figure 1 : Description et projection de la cinétique de la réponse immunitaire à l'infection causée par le coronavirus du syndrome respiratoire aigu sévère 2 (SRAS-CoV-2). Compte tenu que l'expérience à ce jour n'est que de quelques mois, les projections en années, même si elles se fondent sur l'expérience tirée d'autres coronavirus, doivent être présumées spéculatives. Les données sur les réponses IgA (immunoglobuline A) ne sont pas complètes. IgG = immunoglobuline G, IgM = immunoglobuline M. 
appuyer l'utilisation des tests sérologiques aux points de service pour le SRAS-CoV-2 (voir encadré no 2 pour une description de ces trousses) et, au moment d'écrire ces lignes, aucun test sérologique pour le SRAS-CoV-2 aux points de service n'a été approuvé par Santé Canada ${ }^{19}$. Pour pouvoir être utilisés et interprétés aux points de service, ces tests devront générer des résultats qui concordent avec ceux des laboratoires approuvés, et les trousses devront être disponibles en quantité suffisante, en plus d'avoir un rendement conforme aux critères d'assurance de la qualité.

\section{Quels facteurs influent sur l'interprétation des tests sérologiques pour le SRAS-CoV-2?}

\section{Sensibilité, spécificité et prévalence de la maladie}

Le pouvoir discriminant d'un test est évalué sur la base de sa sensibilité et de sa spécificité cliniques. La sensibilité est une mesure de l'aptitude du test à détecter des anticorps dans des sources telles que le sang, le sérum ou le plasma de patients porteurs d'une infection au SRAS-CoV-2 (c.-à-d., résultat vrai positif). La spécificité est une mesure de l'aptitude du test à identifier correctement l'absence d'anticorps chez une personne qui n'a pas été infectée (c.-à-d., résultat vrai négatif). À l'heure actuelle, Santé Canada recommande une spécificité cible de $98 \%$ ou plus, et le minimum requis pour envisager une approbation est de $95 \%{ }^{21}$. La sensibilité et la spécificité sont indissociables du rendement des tests, mais leur valeur prédictive dépend toujours de la prévalence de l'infection au sein d'une population donnée. Par exemple, si la prévalence de la maladie dans la population n'est que de $1 \%$, même un test diagnostique hautement spécifique (spécificité de $99 \%$ ou seulement 1 résultat faux positif sur 100 résultats-patients) prédirait environ 1 résultat faux positif pour chaque résultat vrai positif ${ }^{9,22}$.

Au Canada, la prévalence de base de l'infection au SRASCoV-2 en date du 9 juillet 2020 était estimée à 0,3\%, pour 106805 cas confirmés de COVID-1923. Mais le nombre de Canadiens infectés est probablement beaucoup plus élevé que ne l'indique le nombre de cas confirmés, et ce, pour plusieurs raisons. Les tests actuels sur l'acide nucléique ont une sensibilité modérée et les tests effectués sur les patients infectés peuvent avoir donné des résultats négatifs dans certains cas. En outre, les indications et la couverture des tests ont varié avec le temps et selon les régions; et certaines personnes porteuses du SRASCoV-2 ne montrent aucun symptôme, donc, plusieurs personnes infectées n'ont peut-être pas demandé à subir le test ou n'y étaient pas admissibles. Ailleurs, la prévalence de l'infection asymptomatique mesurée varie grandement selon la population cible testée, le lieu et l'âge des patients ${ }^{14,24-27}$.

Selon nous, dans la plupart des régions canadiennes, la prévalence est probablement suffisamment faible pour que de très petites réductions de la spécificité du test fassent augmenter la proportion de faux positifs rapportés. Cela peut être corrigé a posteriori par des estimations de la prévalence des infections au SRAS-CoV-2 dans la population, mais complique l'application des résultats sérologiques à l'échelle individuelle, un défi que viennent encore compliquer les imprécisions biologiques.
Encadré no 2 : Approches au dépistage des anticorps du coronavirus du syndrome respiratoire aigu sévère 2

\section{Nature des échantillons analysés}

- Les tests énumérés ici sont en général utilisés pour détecter la présence d'anticorps dans le sang.

- Les immunodosages et les tests de neutralisation spécialisés effectués en laboratoire se font sur des échantillons de sang (sérum ou plasma). Les tests aux points de service se font par des piqûres au bout du doigt (ou au talon, selon le cas).

- Les piqûres au bout du doigt peuvent aussi servir pour créer des gouttes de sang séché faciles à manipuler/transporter pour les immunodosages en laboratoire. Les premiers résultats des tests réalisés sur des gouttes de sang séché sont prometteurs, mais les données probantes sont actuellement insuffisantes pour les promouvoir pour le coronavirus du syndrome respiratoire aigu sévère 2 (SRAS-CoV-2). Un peu partout dans le monde, on travaille à leur validation complète en les comparant aux résultats des tests effectués sur des prélèvements sanguins.

- Quant à la fiabilité et à l'interprétation des résultats des recherches d'anticorps dans des échantillons d'autre nature que le sang (p. ex., salive) elles restent à confirmer.

\section{Types de tests}

- Les immunodosages en laboratoire reposent sur des plaques à microtitrage (tests ELISA, enzyme-linked immunosorbent assay) ou sur des immunodosages par chimioluminescence au moyen d'instruments automatisés de grande capacité. Avec la plupart des immunodosages, le signal produit par le test est en corrélation avec le taux d'anticorps détecté dans l'échantillon. Les réactions croisées avec d'autres coronavirus sont rares, mais elles surviennent. Pour les tests en laboratoire, il faut tenir compte des prélèvements et du transport vers le laboratoire. Les immunodosages commerciaux disponibles sont semi-quantitatifs (c.-à-d., qu'ils utilisent un seuil pour déterminer les résultats réactifs [positifs] c. non réactifs [négatifs]).

- Les cassettes de tests à réponse rapide, ou tests de flux latéral, sont souvent mentionnées parmi les tests effectués aux points de service. Ces tests sont généralement faciles à utiliser, ne requièrent qu'une petite quantité de sang (p. ex., piqûre au bout du doigt) sans équipement ni expertise spécialisés en plus de donner les résultats rapidement (10 à 15 minutes) ${ }^{18}$. La plupart permettent de détecter l'immunoglobuline $M$ et l'immunoglobuline $G$ séparément ou ensemble. Toutefois, ces dosages sont qualitatifs, leur rendement varie substantiellement et aucun n'a été approuvé à ce jour par Santé Canada. Ces tests ne sont pas indiqués aux points de service ${ }^{19}$.

- Les tests de neutralisation sont considérés comme la norme pour le dépistage des anticorps en raison de leurs grandes sensibilité et spécificité, mais ils ne sont pas facilement accessibles. Les immunodosages en laboratoire et aux points de service sont des dosages des « anticorps liés » (c.-à-d., qu'ils détectent la fixation des anticorps aux antigènes du SRAS-CoV-2 dans les prélèvements). Quant à eux, les tests de neutralisation mesurent l'inhibition immunitaire de l'entrée du virus dans les cellules in vitro. Ces tests requièrent une expertise spécialisée et des laboratoires de confinement (de niveau 3 pour le SRASCoV-2), et ils ont une capacité limitée. Toutefois, les tests de neutralisation avec pseudovirus peuvent détecter les anticorps anti-SRAS-CoV-2 neutralisants à l'aide de souches virales modifiées génétiquement et peuvent être effectués dans des laboratoires de confinement de niveau 2. 


\section{Corrélation entre anticorps anti-SRAS-CoV-2 et neutralisation virale}

Les dosages sérologiques commerciaux disponibles détectent et déterminent de manière semi-quantitative la quantité d'anticorps liés aux divers antigènes du SRAS-CoV-2. (La quantification peut être utile lorsqu'une hausse des taux suggère une infection récente suivie de séroconversion.) Selon la cible antigénique utilisée, la détection d'anticorps fixés peut être en corrélation avec la détection d'anticorps neutralisants qui, comme on le mentionnait précédemment, bloquent la liaison virale et neutralisent l'infection in vitro (d'où le terme anticorps neutralisants). Les anticorps neutralisants peuvent être plus utiles pour déterminer l'immunité, mais le débat continue quant à leur rôle comme principal mode de protection immunitaire contre le SRAS-CoV-222,28-30. Une réponse à médiation cellulaire, connue pour son rôle clé dans le contrôle viral du SRAS-CoV-1 et du SRMO-CoV ${ }^{31-33}$, serait peut-être plus pertinente. Il faudra donc approfondir la recherche pour évaluer la corrélation entre les dosages commerciaux d'anticorps anti-SRAS-CoV-2 neutralisants, la possibilité de réponses immunitaires à médiation cellulaire dépendantes des anticorps et la séroprotection ${ }^{34,35}$.

\section{Durée de la réponse immunitaire}

Dans les cas bénins et asymptomatiques, les réponses immunitaires peuvent être variables ou ne pas atteindre des taux suffisants pour être détectables au moyen des dosages d'anticorps ${ }^{36}$. La recherche se poursuit sur l'ampleur et la durée des réponses immunitaires dans les infections allant d'asymptomatiques à graves, et dans différentes populations, selon l'âge, le bagage génétique et les comorbidités. Les taux d'anticorps anticoronavirus diminuent avec le temps ${ }^{37,38}$. Mais malgré un déclin des titres, une persistance des anticorps neutralisants mesurables pendant au moins 1 an a été signalée chez des patients qui se sont remis d'un SRMO-CoV ou d'un SRAS$\mathrm{CoV}-1^{39,40}$. Mais des études sur la réponse immunitaire au SRASCoV-2 ont laissé entendre qu'une proportion substantielle de patients asymptomatiques sont IgG-négatifs durant la phase convalescente de l'infection ${ }^{36}$. Et selon de récentes données probantes, les anticorps anti-SRAS-CoV-2 neutralisants diminuent chez les patients convalescents dans les 2 à 3 mois qui suivent l'infection $^{36,41}$. En revanche, une persistance des réponses liées aux lymphocytes $T$ à mémoire pendant plusieurs années a été signalée avec le SRMO-CoV et le SRAS-CoV-132,33; on en déduit que la baisse des titres d'anticorps n'est peut-être pas incompatible avec l'immunité. Le lien entre les anticorps mesurés et la durée de la protection reste donc à déterminer.

\section{Séropositivité et infectiosité}

La présence confirmée d'anticorps ne va pas forcément de pair avec un état non infectieux. En particulier, dans le cas des anticorps non neutralisants, la présence d'anticorps n'empêche pas l'excrétion virale active dans les expectorations ${ }^{42,43}$. Ainsi, des facteurs tels que le déclenchement des symptômes, leur résolution et le nombre de jours suivant leur déclenchement ou leur résolution devraient servir de guides concernant l'infectivité.

\section{Quelles sont les répercussions pour les médecins et les décideurs?}

\section{Tenir compte du rendement des tests}

Les laboratoires devraient veiller à utiliser des tests sérologiques pour le SRAS-CoV-2 dont les fabricants assurent qu'ils ont une sensibilité de $95 \%$ ou plus et une spécificité de $99,5 \%$ ou plus, sur des spécimens obtenus 14 jours ou plus après le déclenchement des symptômes ou un test de recherche d'ARN positif. Les tests sérologiques sont nécessaires dans les milieux de soins pour confirmer qu'ils ont le rendement prévu. Pour une meilleure spécificité dans des populations chez qui la probabilité prétest de la maladie est faible (c.-à-d., faible prévalence dans la communauté), il pourrait être nécessaire d'utiliser des tests orthogonaux afin de soumettre d'emblée à un test de confirmation les patients qui ont un résultat positif à un premier test.

Pour interpréter les résultats sérologiques, les laboratoires pourraient devoir tenir compte du temps écoulé depuis le déclenchement des symptômes ou depuis l'exposition au SRASCoV-2, si l'information est disponible. Les laboratoires cliniques et les médecins demandeurs doivent aussi tenir compte de la probabilité prétest d'un résultat positif ou négatif selon le lieu du test et la population concernée (p. ex., probabilité plus forte d'un test positif dans un hôpital par rapport à un dépistage de la séroprévalence en clinique externe).

Il faudra approfondir la recherche sur le rendement des tests sérologiques auprès d'individus appartenant à différents groupes d'âge à différents intervalles suivant l'exposition pour déterminer la durée de la détectabilité des anticorps après l'infection.

\section{À quel moment et pour qui ces tests?}

Pour l'instant, les tests sérologiques pour le SRAS-CoV-2 devraient servir à guider la réponse de la santé publique face à l'épidémie au Canada plutôt qu'à estimer la sensibilité actuelle ou future des individus à l'infection. Ils devraient être principalement réservés à la recherche clinique et à l'évaluation de la prévalence des infections passées au SRAS-CoV-2 dans la population.

Comme on le mentionnait précédemment, les tests sérologiques ne sont ni adéquats ni appropriés comme outils diagnostiques primaires ou pour confirmer la non-infectivité. En d'autres mots, l'état actuel des connaissances n'appuie pas l'utilisation à grande échelle du dosage des anticorps anti-SRASCoV-2. En l'absence de tests de recherche d'ARN positifs ou de signes de séroconversion (mesurés par une augmentation progressive des taux d'anticorps), la sérologie ne devrait pas être utilisée pour diagnostiquer une infection aiguë au SRAS-CoV-2. En de rares occasions, les tests sérologiques pourraient être utiles comme outils diagnostiques d'appoint lorsque les épreuves moléculaires demeurent négatives en présence d'un fort soupçon clinique et de symptômes persistants ${ }^{7,13,44-46}$. Dans de tels cas, le temps écoulé depuis l'exposition (s'il est connu) ou depuis le déclenchement des symptômes devrait entrer en ligne de compte puisque la séropositivité ne se manifeste que 7 à 14 jours ou plus après le déclenchement des symptômes. 
Encadré no 3 : Rôle de la sérologie dans le syndrome inflammatoire multisystémique présumé, en lien temporel avec la maladie à coronavirus 2019, chez l'enfant et l'adolescent

- Le syndrome inflammatoire multisystémique (SIM) chez l'enfant a été décrit par plusieurs organisations, y compris l'Organisation mondiale de la santé, les Centers for Disease Control and Prevention, le Royal College of Paediatrics and Child Health du Royaume-Uni et la Société canadienne de pédiatrie ${ }^{48-51}$, et la définition repose notamment sur les tests sérologiques de dépistage du coronavirus du syndrome respiratoire aigu sévère 2 (SRAS-CoV-2).

- Des séries de cas ont révélé de forts pourcentages de patients ayant obtenu des résultats positifs au test sérologique de dépistage du SRAS-CoV-2, avec des taux moindres de positivité dans les spécimens de gorge et de selles (test d'amplification en chaîne par polymérase) $)^{52-54}$.

- Plusieurs provinces canadiennes sont en voie d'adopter ces critères et ont classé le syndrome inflammatoire multisystémique chez l'enfant parmi les maladies à déclaration obligatoire qui doivent être signalées aux autorités de santé publique locales ${ }^{55-57}$.

- Malgré l'inclusion des tests sérologiques pour le syndrome inflammatoire multisystémique, leur rendement chez les enfants et chez les patients atteints de SIM sont mal caractérisés.

- Il faudra approfondir la recherche, y compris au moyen d'études de validation des dosages des anticorps anti-SRAS-CoV- 2 chez les enfants et dans les cas de SIM présumés.

Les tests sérologiques peuvent aider à l'évaluation des patients qui ont des manifestations cliniques atypiques, comme des syndromes inflammatoires (p. ex., syndrome inflammatoire multisystémique chez les enfants et les adolescents, orteils COVID ou thrombose inexpliquée) $)^{47,48}$. L'encadré no 3 présente les tests immunologiques dans le contexte du syndrome inflammatoire multisystémique chez les enfants et les adolescents à partir des données probantes actuelles, qui sont limitées ${ }^{13,49-54}$.

\section{Comment présenter les résultats des tests sérologiques?}

Un récent rapport publié au Royaume-Uni ${ }^{58}$ a révélé une variabilité dans l'interprétation clinique des résultats sérologiques relatifs au SRAS-CoV-2, particulièrement en ce qui concerne l'inférence de l'immunité et le statut infectieux des individus. Pour que le message soit cohérent et pour éviter les erreurs d'interprétation des résultats des tests sérologiques, il faudra voir à l'harmonisation des rapports des laboratoires et à une communication proactive de la part du personnel des laboratoires, des microbiologistes médicaux et des infectiologues. L'encadré no 4 présente des suggestions pour le libellé des rapports d'interprétation qui peuvent provisoirement être utilisées par les laboratoires cliniques et de santé publique dans leurs communications sur la sérologie du SRAS-CoV-2.

\section{Utilisations potentielles de la sérologie du SRAS-CoV-2 pour la santé publique et la recherche}

À l'heure actuelle, sur la base des données probantes analysées, les résultats des tests sérologiques ne devraient pas servir à guider la prise de décisions à l'échelle des individus pour les
Encadré no 4 : Suggestions de libellés pour les laboratoires cliniques qui rapportent les résultats (incluant commentaires en annexe)

\section{Rapports de résultats positifs/réactifs}

- La présence d'anticorps anticoronavirus du syndrome respiratoire aigu sévère 2 (SRAS-CoV-2) indique une infection en cours ou résorbée. Des résultats faux positifs peuvent être obtenus en raison notamment d'anticorps à réaction antigénique croisée. Pour l'instant, on ignore si les anticorps donnent lieu à une immunité protectrice et quel en serait la durée.

- La présence d'anticorps anti-SRAS-CoV-2 ne devrait pas servir à inférer le statut infectieux ou l'immunité d'un individu.

\section{Rapport de résultats négatifs/non réactifs}

- Les résultats non réactifs ne permettent pas d'écarter une infection aiguë ou passée au SRAS-CoV-2.

- L'absence d'anticorps anti-SRAS-CoV-2 ne devrait pas servir pour inférer le statut infectieux ou l'immunité d'un individu

\section{Rapport de résultats non concluants/indéterminés}

- Un résultat non concluant ne permet ni d'infirmer ni de confirmer une infection passée au SRAS-CoV-2.

mesures de contrôle des infections, y compris l'utilisation de l'équipement de protection personnelle, le moment du retour au travail ou les politiques locales de distanciation physique.

Par contre, des études de séroprévalence du SRAS-CoV-2 pourraient servir à estimer les taux d'exposition et la transmission géographique du virus dans les communautés et les populations, de même que dans les établissements, les milieux de travail ou les ménages au fil du temps. Cette information pourrait servir à modéliser l'épidémie et guider les politiques de santé publique pour les planificateurs des programmes de vaccination afin d'établir les priorités et pour les professionnels de la santé publique sur la ligne de front pour identifier les communautés ou environnements ayant été minimalement exposés au SRAS-CoV-2 et pouvant, par conséquent, être plus sujets à une propagation rapide. Au carrefour entre les applications cliniques et de santé publique, alors que le rôle diagnostique des tests immunologiques est strictement complémentaire, des études de séroprévalence pourraient servir à la recherche de contacts en présence de tests d'ARN non concluants.

Des études de séroprévalence longitudinales pourraient fournir des renseignements sur la nature et la durée des réponses immunitaires chez les patients porteurs d'une infection avérée. Le but de telles études serait de déterminer si le fait d'avoir souffert de la COVID-19 et d'être séropositif protège contre la réinfection; les tests sérologiques spécialisés, comme les dosages d'anticorps neutralisants, seront particulièrement utiles dans ce contexte. De même, le dépistage sérologique sur les dons de sang pourrait révéler quels échantillons sanguins renferment des taux adéquats d'anticorps neutralisants pour en permettre l'utilisation dans le cadre d'essais randomisés et contrôlés visant à vérifier l'efficacité du traitement à base de plasma convalescent dans les cas graves de COVID-19.

La recherche sur l'efficacité des vaccins anti-SRAS-CoV-2 pourrait utiliser les résultats des tests sérologiques comme marqueurs de l'immunité dans des études de cohorte sur les corrélats de la protection et du risque de réinfection. 


\section{Conclusion}

Compte tenu du flou qui entoure les mesures et l'interprétation des résultats, à quelques exceptions près, les épreuves sérologiques pour le SRAS-CoV-2 sont peu indiquées. Elles seront utiles dans quelques contextes pour la recherche et pour l'établissement de politiques de santé publique, mais sur la base des données probantes actuelles, elles ne devraient pas être déployées pour une utilisation clinique à grande échelle. Le soin qui sera apporté à l'interprétation et à la diffusion des rapports sur les résultats des tests est important. L'état actuel des connaissances ne permet pas de faire des inférences définitives quant à l'immunité et la probabilité de réinfection à partir des résultats des tests sérologiques; ces derniers ne peuvent donc pas éclairer la prise de décisions à l'échelle des individus, dans le but de réduire l'exposition professionnelle, conseiller le recours à l'équipement de protection personnelle, recommander la distanciation physique ou formuler des avis sur les voyages internationaux.

\section{Références}

1. Garg S, Kim L, Whitaker M, et al. Hospitalization rates and characteristics of patients hospitalized with laboratory-confirmed coronavirus disease 2019 - COVID-NET, 14 States, March 1-30, 2020. MMWR Morb Mortal Wkly Rep 2020;69:458-64.

2. Woloshin S, Patel N, Kesselheim AS. False negative tests for SARS-CoV-2 infection challenges and implications. N Eng J Med 5 juin 2020. [Cyberpublication avant impression]. doi: 10.1056/NEJMp2015897.

3. To KK-W, Tsang OT-Y, Leung W-S, et al. Temporal profiles of viral load in posterior oropharyngeal saliva samples and serum antibody responses during infection by SARS-CoV-2: an observational cohort study. Lancet Infect Dis 2020;20:565-74.

4. Bullard J, Dust K, Funk D, et al. Predicting infectious SARS-CoV-2 from diagnostic samples. Clin Infect Dis 22 mai 2020. [Cyberpublication avant impression]. doi: 10.1093/cid/ciaa638.

5. Zhou B, She J, Wang Y, et al. The duration of viral shedding of discharged patients with severe COVID-19. Clin Infect Dis 17 avril 2020. [Cyberpublication avant impression]. doi: 10.1093/cid/ciaa451.

6. Sun B, Feng Y, Mo X, et al. Kinetics of SARS-CoV-2 specific IgM and IgG responses in COVID-19 patients. Emerg Microbes Infect 2020;9:940-8.

7. Zhang G, Nie S, Zhang Z, et al. Longitudinal change of SARS-CoV2 antibodies in patients with COVID-19. J Infect Dis 2 mai 2020 [Cyberpublication avant impression]. doi: 10.1093/infdis/jiaa229.

8. Kofler N, Baylis F. Ten reasons why immunity passports are a bad idea. Nature 2020;581:379-81.

9. Weinstein MC, Freedberg KA, Hyle EP, et al. Waiting for certainty on COVID-19 antibody tests - At what cost? N Eng J Med 5 juin 2020. [Cyberpublication avant impression]. doi: 10.1056/NEJMp2017739.

10. Jiang S, Hillyer C, Du L. Neutralizing antibodies against SARS-CoV-2 and other human coronaviruses [erratum publié dans Trends Immunol 2020;41:545]. Trends Immunol 2020;41:355-9.

11. Wu Y, Wang F, Shen C, et al. A noncompeting pair of human neutralizing antibodies block COVID-19 virus binding to its receptor ACE2. Science 2020; 368:1274-8.

12. Ju B, Zhang Q, Ge J, et al. Human neutralizing antibodies elicited by SARSCoV-2 infection. Nature 26 mai 2020. [Cyberpublication avant impression]. doi: 10.1038/s41586-020-2380-z

13. Interim guidelines for COVID-19 antibody testing: interim guidelines for COVID19 antibody testing in clinical and public health settings. Centers for Disease Control and Prevention. Accessible ici : www.cdc.gov/coronavirus/2019-ncov/ lab/resources/antibody-tests-guidelines.html (consulté le 5 juin 2020).

14. Bryan A, Pepper G, Wener MH, et al. Performance characteristics of the Abbott Architect SARS-CoV-2 IgG assay and seroprevalence in Boise, Idaho. J Clin Microbiol 7 mai 2020. [Cyberpublication avant impression]. doi: 10.1128/ JCM.00941-20.

15. Yongchen Z, Shen $\mathrm{H}$, Wang $\mathrm{X}$, et al. Different longitudinal patterns of nucleic acid and serology testing results based on disease severity of COVID-19 patients. Emerg Microbes Infect 2020;9:833-6.
16. Liu Z-L, Liu Y, Wan L-G, et al. Antibody profiles in mild and severe cases of COVID-19. Clin Chem 10 juin 2020 [Cyberpublication avant impression]. doi: 10.1093/clinchem/hvaa137.

17. Long Q-X, Liu B-Z, Deng H-J, et al. Antibody responses to SARS-CoV-2 in patients with COVID-19. Nat Med 2020;26:845-8.

18. Yip PM, Venner AA, Shea J, et al. Point-of-care testing: a position statement from the Canadian Society of Clinical Chemists. Clin Biochem 2018;53:156-9.

19. Groupe de travail sur les infections à virus respiratoires. Énoncé du Réseau des laboratoires de santé publique du Canada (RLSPC) sur les tests sérologiques au point de soins dans le cadre de la COVID-19. Association canadienne de microbiologie clinique et des maladies infectieuses; 2020. Accessible ici : www. cacmid.ca/wp-content/uploads/ReVi-Position-Statement-on-POC-Serology -v1.00.pdf (consulté le 30 juin 2020).

20. Instruments médicaux autorisés pour les utilisations liées à la COVID-19 : Liste d'instruments de dépistage autorisés. Ottawa : Santé Canada; modifié le 14 juillet 2020. Accessible ici : https://www.canada.ca/fr/sante-canada/services/ medicaments-produits-sante/covid19-industrie/instruments-medicaux/autorises/ liste.html (consulté le 6 juillet 2020).

21. Instruments de dépistage sérologique de la COVID-19: Avis concernant les valeurs de sensibilité et de spécificité. Ottawa: Santé Canada. Le 24 juin 2020, modifié le 25 juin. Accessible ici : https://www.canada.ca/fr/sante-canada/services/ medicaments-produits-sante/covid19-industrie/instruments-medicaux/depistage/ serologique/avis-valeurs-sensibilite-specificite.html (consulté le 8 juillet 2020).

22. Bryant JE, Azman AS, Ferrari MJ, et al. Serology for SARS-CoV-2: apprehensions, opportunities, and the path forward. Sci Immunol 2020;5:eabc6347.

23. Maladie à coronavirus (COVID-19) : mise à jour sur l'éclosion. Ottawa : Agence de la santé publique du Canada; modifié le 11 juillet 2020. Accessible ici : https://www.canada.ca/fr/sante-publique/services/maladies/2019-nouveau -coronavirus.html (consulté le 9 juillet 2020).

24. Mizumoto K, Kagaya K, Zarebski A, et al. Estimating the asymptomatic proportion of coronavirus disease 2019 (COVID-19) cases on board the Diamond Princess cruise ship, Yokohama, Japan, 2020. Euro Surveill 2020;25:2000180.

25. Nishiura $H$, Kobayashi $\mathrm{T}$, Suzuki $A$, et al. Estimation of the asymptomatic ratio of novel coronavirus infections (COVID-19). Int J Infect Dis 2020;94:154-5.

26. Qiu H, Wu J, Hong $L$, et al. Clinical and epidemiological features of 36 children with coronavirus disease 2019 (COVID-19) in Zhejiang, China: an observational cohort study. Lancet Infect Dis 2020;20:689-96.

27. Portail des connaissances internationales sur les tests sérologiques: SeroTracker. Montréal : Groupe de travail sur l'immunité face à la COVID-19. Accessible ici : https://www.covid19immunitytaskforce.ca/fr/recherche/portail-de -connaissances-internationales-sur-les-tests-serologiques/ (Consulté le 20 juin 2020).

28. Cao Y, Su B, Guo X, et al. Potent neutralizing antibodies against SARS-CoV-2 identified by high-throughput single-cell sequencing of convalescent patients' B cells. Cell 2020;182:73-84.e16.

29. Chandrashekar A, Liu J, Martinot AJ, et al. SARS-CoV-2 infection protects against rechallenge in rhesus macaques. Science 20 mai 2020. [Cyberpublication avant impression]. doi: 10.1126/science.abc4776.

30. Kirkcaldy RD, King BA, Brooks JT. COVID-19 and postinfection immunity: limited evidence, many remaining questions. JAMA 11 mai 2020. [Cyberpublication avant impression]. doi: 10.1001/jama.2020.7869.

31. Channappanavar R, Zhao J, Perlman S. T cell-mediated immune response to respiratory coronaviruses. Immunol Res 2014;59:118-28.

32. Ng O-W, Chia A, Tan AT, et al. Memory T cell responses targeting the SARS coronavirus persist up to 11 years post-infection. Vaccine 2016;347:2008-14.

33. Channappanavar R, Fett C, Zhao J, et al. Virus-specific memory CD8 T cells provide substantial protection from lethal severe acute respiratory syndrome coronavirus infection. J Virol 2014;88:11034-44.

34. Ni L, Ye F, Cheng M-L et coll. Detection of SARS-CoV-2-specific humoral and cellular immunity in COVID-19 convalescent individuals. Immunity 2020;52:971-7.e3.

35. Tay MZ, Poh CM, Rénia L, et al. The trinity of COVID-19: immunity, inflammation and intervention. Nat Rev Immunol 2020;20:363-74.

36. Long Q-X, Tang X-J, Shi Q-L, et al. Clinical and immunological assessment of asymptomatic SARS-CoV-2 infections. Nat Med 18 juin 2020 [Cyberpublication avant impression]. doi: 10.1038/s41591-020-0965-6.

37. Kellam P, Barclay W. The dynamics of humoral immune responses following SARS-CoV-2 infection and the potential for reinfection. J Gen Virol 20 mai 2020. [Cyberpublication avant impression]. doi: 10.1099/jgv.0.001439.

38. Lin Q, Zhu L, Ni Z, et al. Duration of serum neutralizing antibodies for SARS-CoV-2: Lessons from SARS-CoV infection. J Microbiol Immunol Infect 25 mars 2020. [Cyberpublication avant impression]. doi: 10.1016/j.jmii.2020.03.015.

39. Cao W-C, Liu W, Zhang P-H, et al. Disappearance of antibodies to SARSassociated coronavirus after recovery. N Engl J Med 2007;357:1162-3. 
40. Choe PG, Perera RAPM, Park WB, et al. MERS-CoV antibody responses 1 year after symptom onset, South Korea, 2015. Emerg Infect Dis 2017;23:1079-1084.

41. Payne DC, Smith-Jeffcoat SE, Nowak G, et al. SARS-CoV-2 infections and serologic responses from a sample of U.S. Navy service members - USS Theodore Roosevelt, April 2020. MMWR Morb Mortal Wkly Rep 2020;69:714-721.

42. Wölfel R, Corman VM, Guggemos W, et al. Virological assessment of hospitalized patients with COVID-2019. Nature 2020;581:465-69.

43. Atkinson B, Petersen E. SARS-CoV-2 shedding and infectivity. Lancet 2020;395:1339-40.

44. ISDA COVID-19 antibody testing primer. Arlington (VA): Infectious Diseases Society of America; mis à jour le 4 mai 2020. Accessible ici : www.idsociety.org/ globalassets/idsa/public-health/covid-19/idsa-covid-19-antibody-testing -primer.pdf (consulté le 20 juin 2020).

45. Guo L, Ren L, Yang S, et al. Profiling early humoral response to diagnose novel coronavirus disease (COVID-19). Clin Infect Dis 21 mars 2020. [Cyberpublication avant impression]. doi: 10.1093/cid/ciaa310.

46. Zhao R, Li M, Song H, et al. Early detection of SARS-CoV-2 antibodies in COVID19 patients as a serologic marker of infection. Clin Infect Dis $1^{\mathrm{er}}$ mai 2020. [Cyberpublication avant impression]. doi: 10.1093/cid/ciaa523.

47. Feldstein LR, Rose EB, Horwitz SM, et al. Multisystem inflammatory syndrome in U.S. children and adolescents. N Engl J Med 29 juin 2020 [Cyberpublication avant impression]. doi: 10.1056/NEJMoa2021680.

48. Le syndrome inflammatoire multisystémique de l'enfant ayant un lien temporel avec la COVID-19 [document de principes]. Ottawa : Société canadienne de pédiatrie; 2016. Accessible ici : https://www.cps.ca/fr/documents/position/ SIME (consulté le 7 juillet 2020).

49. Whittaker E, Bamford A, Kenny J, et al. Clinical characteristics of 58 children with a pediatric inflammatory multisystem syndrome temporally associated with SARS-CoV-2. JAMA 8 juin 2020. [Cyberpublication avant impression]. doi: 10.1001/jama.2020.10369

50. Multisystem inflammatory syndrome in children and adolescents temporally related to COVID-19 [scientific brief]. Genève : Organisation mondiale de la santé. Le 15 mai 2020. Accessible ici : www.who.int/news-room/commentaries /detail/multisystem-inflammatory-syndrome-in-children-and-adolescents-with -covid-19 (consulté le 20 juin 2020)

51. National Center for Immunization and Respiratory Diseases (NCIRD). Information for healthcare providers about multisystem inflammatory syndrome in children (MIS-C): case definition for MIS-C. Atlanta: Centers for Disease Control and Prevention; révisé le 29 mai 2020. Accessible ici : www.cdc.gov/mis-c/hcp/ (consulté le 15 juin 2020).

52. Toubiana J, Poirault C, Corsia A, et al. Kawasaki-like multisystem inflammatory syndrome in children during the covid-19 pandemic in Paris, France: prospective observational study. BMJ 2020;369:m2094.

53. Belhadjer Z, Méot M, Bajolle F, et al. Acute heart failure in multisystem inflammatory syndrome in children (MIS-C) in the context of global SARS-CoV-2 pandemic. Circulation 17 mai 2020. [Cyberpublication avant impression]. doi: 10.1161/CIRCULATIONAHA.120.048360.

54. Multisystem inflammatory syndrome in children (MIS-C) associated with coronavirus disease 2019 (COVID-19). Centers for Disease Control and Prevention Accessible ici : https://emergency.cdc.gov/han/2020/han00432.asp (consulté le $1^{\mathrm{er}}$ juillet 2020).

55. Public health disease management guidelines: multisystem inflammatory syndrome in children (MIS-C). Edmonton : Gouvernement de l'Alberta; mis à jour le 31 mai. Accessible ici : https://open.alberta.ca/dataset/7332b06a-515e-4194 -8ffa-a80e4fc0ea3b\#summary (consulté le 22 juin 2020).

56. Multisystem inflammatory syndrome in children (MIS-C) temporally associated with COVID-19: guidance for clinicians in B.C. Vancouver: BC Centre for Disease Control; 2020. Accessible ici : www.bccdc.ca/Health-Professionals-Site/ Documents/COVID19_MIS-C_ClinicianGuidance.pdf (consulté le 22 juin 2020).

57. COVID-19-Ce que nous savons jusqu'à présent sur... une affection apparentée à la maladie de Kawaski. Toronto : Santé publique Ontario. Accessible ici : www. publichealthontario.ca/-/media/documents/ncov/covid-wwksf/2020/05/what-we -know-kawasaki-disease-like-illness.pdf?la=fr (consulté le 22 juin 2020).

58. Bermingham WH, Wilding T, Beck S, et al. SARS-CoV-2 serology: Test, test, test, but interpret with caution! Clin Med (Lond) 2 juin 2020. [Cyberpublication avant impression]. doi: 10.7861/clinmed.2020-0170.
Intérêts concurrents : Paul Van Caeseele est directeur du laboratoire provincial de santé publique de Cadham (Santé Manitoba). Mel Krajden a reçu des subventions versées à son établissement par Roche, Hologica et Siemens. Aucun autre intérêt concurrent déclaré. Pour les intérêts concurrents des membres des 5 groupes d'auteurs, voir annexe 1, accessible ici https://www.cmaj.ca/lookup/doi/10.1503/cmaj.201588/tab-related-content

Cet article a été révisé par des pairs.

Affiliations : Laboratoire provincial de santé publique Cadham (Van Caeseele), Winnipeg, Man.; Dynacare (Bailey), Brampton, Ont.; Division d'infectiologie pédiatrique (Forgie), Département de pédiatrie, Université de l'Alberta, Edmonton, Alta.; Alberta Precision Laboratories (Dingle), Edmonton, Alta.; et Département de pathologie et de médecine de laboratoire (Krajden), Université de la Colombie-Britannique, Vancouver, C.-B.

Collaborateurs : Tous les auteurs ont contribué à la conception et à la modélisation de la revue; ils ont révisé le manuscrit, qui a été d'abord ébauché par Dana Bailey et Mel Krajden pour ce qui est du contenu intellectuel important; ils ont donné leur approbation finale à la version devant être publiée et assument l'entière responsabilité de tous les aspects du travail. Paul Van Caeseele est coprésident du Réseau canadien des laboratoires de santé publique. Dana Bailey est auteure de correspondance désignée pour le groupe de travail sur la COVID-19 de la Société canadienne des clinico-chimistes. Sarah Forgie est présidente de l'Association pour la microbiologie médicale et l'infectiologie Canada. Tanis Dingle est présidente de l'Association canadienne de microbiologie clinique et des maladies infectieuses. Mel Krajden est responsable du Comité pour les tests du Groupe de travail sur l'immunité face à la COVID-19.

Membres du Réseau canadien des laboratoires de santé publique : Mike Drebot $\mathrm{PhD}^{\star}$, Jean Longtin MD*, Tim Booth PhD† (Laboratoire national de microbiologie), Graham Tipples PhD*, Jamil N. Kanji MD† (Alberta), Inna Sekirov MD PhD*, Muhammad Morshed PhD†, Paul N. Levett PhD DSc $†$ (Colombie-Britannique), Paul Van Caeseele MD* (auteur de correspondance RLSPC), Derek Stein PhD† (Manitoba), Richard Garceau MD* (Nouveau-Brunswick), Lei Jiao MD PhD* (Terre-
Neuve et Labrador), Todd F. Hatchette MD*, Jason LeBlanc PhD† (NouvelleÉcosse), Julianne Kus PhD*, Jonathan Gubbay MBBS MMedSc† (Ontario), Greg J. German MD PhD†, Jason Robinson PhD† (île-du-Prince-Édouard), Michel Roger MD PhD* (Québec), Amanda Lang PhD* (Saskatchewan).; *auteurs, †collaborateurs.

Membres de la Société canadienne des clinico-chimistes (Groupe de travail COVID-19) : Auteurs : Dana Bailey MSc PhD (auteure de correspondance SCCC), Danijela Konforte PhD (Président du groupe d'intérêt COVID-19 de la SCC), Vilte E. Barakauskas PhD, Vathany Kulasingam PhD, Paul M. Yip PhD; collaborateurs : Lori Beach PhD, Ivan Blasutig PhD, Peter Catomeris PhD, Mari L. DeMarco PhD, Yanping Gong MD PhD, Peter A. Kavsak PhD, Ed Randell PhD, Julie Shaw, PhD, Jennifer Taher PhD, Nicole White-Al Habeeb PhD.

Membres de l'Association pour la microbiologie médicale et l'infectiologie Canada : Auteurs : Sarah E. Forgie MD MÉd (auteure de correspondance); collaborateurs : Isabelle Chiu MD, Yves Longtin MD, LeeAnne M. Luft MD MSc, Shariq Haider MD, Susy Hota MD MSc, Philippe LagaceWiens MD, Kelly MacDonald MD, Andrew Simor MD, Karina Top MD MS, Terence C. Wuerz MD, Deborah Yamamura MD.

Membres de l'Association canadienne de microbiologie clinique et des maladies infectieuses : Auteurs : Tanis C. Dingle PhD (auteure de correspondance), Carmen L. Charlton PhD, Samir Patel PhD, Prameet Sheth PhD, Vanessa Tran PhD; collaborateurs : Ramzi Fattouh PhD, Danielle Brabant-Kirwan PhD, Rob Kozak PhD, Chris Lowe MSc MD, Esther Nagai BAA M Bibl, Valentina Russell BSc M Bibl.

Membres du Groupe de travail sur l'immunité face à la COVID-19 : Auteurs : Mel Krajden MD (auteur de correspondance), C. David Naylor MD PhD (coordonnateur éditorial), Timothy G. Evans PhD MD, Catherine A. Hankins MD, James D. Kellner MD, Allison D. McGeer MD, Caroline Quach-Thanh MD; collaborateurs : James Talbot MD PhD, Gina Ogilvie MD MSc, Vivek Goel MD MSc MS, Scott Halperin MD, Jutta Preiksaitis MD.

Correspondance : Mel Krajden, Mel.Krajden@bccdc.ca 